\title{
Non-commutative moduli spaces of topological D-branes
}

\author{
Calin Iuliu Lazaroiu ${ }^{1, *}$ \\ ${ }^{1}$ Department of Mathematics \\ Trinity College Dublin \\ Dublin 2, Ireland \\ calin@maths.tcd.ie
}

Key words topological strings, noncommutative geometry

\begin{abstract}
We give a general construction of extended moduli spaces of topological D-branes as non-commutative algebraic varieties. This shows that noncommutative symplectic geometry in the sense of Kontsevich arises naturally in String Theory.
\end{abstract}

\section{Introduction}

The moduli space of topological D-branes provides a first approximation to the quantum geometry seen by open strings. Because the algebra of boundary operators on a topological open string worldsheet is noncommutative, one tests quantum geometry with a noncommutative probe, so one expects a noncommutative description of the relevant moduli space.

In the traditional approach to D-brane geometry, one starts by fixing a Chan-Paton representation $\Gamma$ and constructs a commutative moduli space which depends markedly on $\Gamma$. This dependence makes it difficult to identify the deeper structure which lies behind particular examples. The task of extracting the latter requires a universal formulation of the deformation problem for topological D-brane systems, which describes boundary deformations in a representation-agnostic manner. In [1], we showed how one can carry out this task starting from the most basic conditions on topological string amplitudes, known as the $A_{\infty}$ constraints. In fact, we carried out this analysis for a general system containing a finite number of Dbranes. Using an algebraic formulation of such systems, we showed that topological boundary amplitudes can be packed into a 'noncommutative generating function' $W$ which generalizes the generating function considered in [4] (itself a generalization of the D-brane superpotential of [3]). Formally, $W$ is an element of the zero'th (relative) Karoubi complex of a certain associative superalgebra $A$, and can also be viewed as a linear combination of necklaces on a superquiver. Moreover, the boundary topological metrics of the system induce a noncommutative symplectic form on $A$ in the sense of [2], which we denote by $\omega$. It was shown in [1] that $W$ is subject to certain constraints, which naturally encode a series of complicated algebraic conditions on the tree-level scattering amplitudes between the open topological strings present in the system. Using these constraints, one can recover $\omega$ from $W$, which means that $W$ suffices to completely characterize tree-level dynamics. The construction of the extended moduli space proceeds in algebraic manner, by considering the invariant part $(A / J)^{\mathcal{G}}$ of the quotient of $A$ through the 'noncommutative critical ideal' $J$ of $W$ under the action of a certain group $\mathcal{G}$ which encodes all autoequivalences of the collection of boundary amplitudes. This algebraic construction has a non-commutative-geometric interpretation in which $A$ is viewed as the noncommutative coordinate ring of a noncommutative affine space $\mathbb{A}$, allowing

* $\quad$ E-mail: calin@maths.tcd.ie 
one to view $W$ as a noncommutative function (in the sense of [2]) defined on that space. Then $A / J$ is the noncommutative coordinate ring of the 'critical locus' $\mathcal{Z}$ of $W$, viewed as a subspace of $\mathbb{A}$, while $(A / J)^{\mathcal{G}}$ is the coordinate ring of the (noncommutative) extended moduli space of interest, which we will denote by $\mathcal{M}$.

\section{The $A_{\infty}$ structure of finite topological D-brane systems}

It is well-known that scattering amplitudes of open strings can be encoded by a series of multilinear maps (the so called boundary string products) defined on the space $E$ of boundary operators and taking values $E$. As explained in detail in [4] (see also [7-9]), the hierarchy of contact terms arising when two boundary operators approach each other is constrained by the geometry of the natural compactification of the moduli space of disks with boundary punctures. As a consequence, the boundary scattering products of a topological open string theory are subject to a countable series of constraints, which turn out to coincide with the algebraic conditions defining an $A_{\infty}$ algebra [5], a fact anticipated in [10] in the context of open bosonic string field theory. Generalizing this observation to systems of topological D-branes leads to the following conclusion:

The collection of all (integrated) boundary and boundary-condition changing tree-level amplitudes of a topological string theory forms a weak, cyclic and unital $A_{\infty}$ category.

Above, the worldsheet model of a topological string theory is assumed to be a (possibly massive) deformation of a topological worldsheet theory obtained by twisting a model with $\mathrm{N}=2$ superconformal invariance. Indeed, the proof of [4] relies on the existence of a point in the topological deformation space at which the worldsheet model is a topological conformal field theory. We refer the reader to [4] for a precise formulation of the assumptions. A standard example are the topological open string theories associated with topological sigma models $[6,23]$.

\section{Encoding the $A_{\infty}$ structure}

As usually formulated, the structure of a weak, cyclic and unital $A_{\infty}$ category is rather complicated (see Appendix A to [1]) and does not easily allow for general insights. When the system contains a finite number of topological D-branes, of the results of [1] is an equivalent description of this structure in a language which clarifies its non-commutative geometric meaning. For this, we start with the observation that a finite collection $\mathcal{Q}_{0}$ of D-branes - viewed as an abstract finite set- allows us to define a semisimple commutative algebra $R$ via the following expression:

$$
R:=\oplus_{u \in \mathcal{Q}_{0}} \mathbb{C} \epsilon_{u}
$$

where $\epsilon_{u}$ are mutually commuting idempotents:

$$
\epsilon_{u} \epsilon_{v}=\delta_{u v} \epsilon_{u} .
$$

Noticing that $R$ is unital with unit $1_{R}=\sum_{u \in \mathcal{Q}_{0}} \epsilon_{u}$, we find that the field of complex numbers embeds into $R$ via:

$$
\mathbb{C} \equiv \mathbb{C} 1_{R} \subset R
$$

For each ordered pair of D-branes $(u, v)$, we have a super-vector space $E_{u v}$ spanned by all topological boundary operators which change the boundary condition from $u$ to $v$ (the case $u=v$ corresponds to the space of boundary operators for a topological open string whose endpoints lie on the D-brane $u$ ). The total boundary space is defined as the super-vector space $E=\oplus_{u, v \in \mathcal{Q}_{0}} E_{u v}$. A useful observation is that giving such a decomposition amounts to giving an $R$-superbimodule structure on $E$. Thus the 'boundary sector decomposition' of the system is encoded by an $R$-superbimodule $E$ (which in most physical examples is finite-dimensional as a complex vector space). 
The next step is to consider the tensor algebra $A=T_{R} E[1]^{\mathrm{v}}$ of the parity changed dual of this superbimodule. This is an $R$-superalgebra since it contains $R$ as a subalgebra in its even subspace. Applying a super-extension of a basic construction to this superalgebra allows us to construct its (relative) differential envelope $\Omega_{R} A$, a $\mathbb{Z} \times \mathbb{Z}_{2}$-graded associative algebra whose elements are known as noncommutative differential (super)forms with coefficients in $A$. The envelope $\Omega_{R} A$ is endowed with a differential $d$, which in the conventions of [1] has bi-degree $(1,0)$. The relative Karoubi complex of $A$ is the quotient $C_{R}(A):=\Omega_{R} A /\left[\Omega_{R} A, \Omega_{R} A\right]$ of $A$ via the subspace $\left[\Omega_{R} A, \Omega_{R} A\right]$ defined as the image of the graded commutator map [., .] : $\Omega_{R} A \times \Omega_{R} A \rightarrow \Omega_{R} A$. This is a $\mathbb{Z} \times \mathbb{Z}_{2}$-graded complex, whose differential $\bar{d}$ is induced from $\Omega_{R} A$. The elements of the $\mathbb{Z}$-homogeneous components $C_{R}^{n}(A)$ are called non-commutative differential $n$-superforms with coefficients in A. According to [2], a non-commutative symplectic form on $A$ is an element $\omega \in C_{R}^{2}(A)$ which satisfies a certain non-degeneration condition (see [1] for a precise formulation of this property). This noncommutative symplectic form has a $\mathbb{Z}_{2}$-degree $\tilde{\omega}$, which is one of the data of the underlying topological string theory. As explained in [1], $\omega$ induces a Lie superbracket on the parity-changed space $C_{R}^{0}(A)[\tilde{\omega}]$. We are ready to formulate ${ }^{1}$ one of the results of [1].

Giving a cyclic $A_{\infty}$ category with finite-dimensional morphism spaces and having a finite number of objects is equivalent to giving the following data:

(1) A finitely-generated semisimple commutative algebra $R$ over the complex numbers.

(2) An $R$-superbimodule $E$ which has finite dimension as a vector space over $\mathbb{C} \subset R$

(3) A noncommutative symplectic form $\omega \in C_{R}^{2}(A)$ on the tensor algebra $A:=T_{R} E$, whose $\mathbb{Z}_{2}$-degree we denote by $\tilde{\omega}$.

(4) An element $W \in C_{R}^{0}(A)$, of $\mathbb{Z}_{2}$-degree $\tilde{\omega}+1$, which satisfies the condition $\{W, W\}=0$, where $\{.,$.$\} is the Kontsevich superbracket defined by \omega$.

Using the non-commutative geometric interpretation of [2], this characterization relates finite D-brane systems to a non-commutative version of the QP-manifolds of [19]

Unitality of the weak $A_{\infty}$ category of boundary string products can also be encoded in this language. For this purpose, one notices that any vector $x \in E$ defines a linear operator $\delta_{x}: C_{R}^{0}(A) \rightarrow A$, which we shall call the (left) cyclic derivative along $x$ (indeed, it is shown in [1] that this is a generalization of the cyclic derivatives of $[13,14]$ ). Then a result of [1] characterizes unitality of the weak $A_{\infty}$ category of boundary string products as the requirement that there exists an even central element $\lambda$ of $E$ (called the total $A_{\infty}$ unit) such that $\delta_{\lambda} W$ belongs to a certain subspace of $A$, the so-called subspace of noncommutative moment maps. One can also show that a noncommutative generating function satisfying this property can be used to reconstruct the noncommutative symplectic form $\omega$.

\section{The extended noncommutative moduli space}

The (extended) noncommutative moduli space of the finite topological D-brane system can be cosntructed follows. First, one defines the noncommutative critical ideal $J$ of $W$ to be the two-sided ideal generated by all cyclic derivatives $\delta_{x} W$, where $x$ runs over $E$. Since $\delta_{x}$ depends linearly on $x$ (see [1]), this ideal is finitely generated:any basis $e_{i}$ of $E$ gives a system of generators $\delta_{e_{i}} W$. Further, one considers the group $\mathcal{G}$ formed by all unital autoequivalences of the underlying weak $A_{\infty}$ category which preserve the cyclic structure given by the boundary topological metrics. As shown in [1], $\mathcal{G}$ can also be described as the group formed by those superalgebra automorphisms of $A$ which preserve the noncommutative symplectic form $\omega$ and obey a certain splitting property with respect to the $A_{\infty}$ unit $\lambda$. Using these objects, we consider the invariant subalgebra $(A / J)^{\mathcal{G}}$ of the quotient $A / J$.

To interpret this geometrically, we follow [2] in viewing $A$ as the noncommutative coordinate ring of a noncommutative symplectic space $\mathbb{A}$. With this interpretation, the quotient $A / J$ is the noncommutative coordinate ring of a noncommutative subspace $\mathcal{Z}$ of $\mathbb{A}$, and the group $\mathcal{G}$ acts on $\mathbb{A}$ via symplectomorphisms. The algebra of invariants $(A / J)^{\mathcal{G}}$ is taken to describe the quotient of $\mathcal{Z}$ through this action of

\footnotetext{
1 This is also derived in [20] in the particular case when the left and right $R$-module structures on $E$ agree.
} 
$\mathcal{G}$, which defines the noncommutative extended moduli space $\mathcal{M}$. With this interpretation, $(A / J)^{\mathcal{G}}$ is the noncommutative coordinate ring of the desired extended noncommutative moduli space.

\section{Representations}

It can be shown that Chan-Paton representations of the underlying open strings (which are specified by picking multiplicities for each $D$-brane in the system) amount to representations of the tensor superalgebra $A$. Using this relation, one finds that the extended version of the commutative moduli space considered in traditional analyses of the moduli space of D-brane systems arise as the collection 'matrix-valued' points of $\mathcal{M}$. Therefore, the noncommutative moduli space $\mathcal{M}$ plays the role of a universal moduli space which encodes all choices of D-brane multiplicity. This gives a physical realization of the ideas of Kontsevich [2], which were extensively developed in $[11,12,15-18,24]$.

\section{On the computation of $\mathcal{M}$}

It is in general straightforward to describe $\mathcal{Z}$ explicitly upon using standard algebraic methods. The computation of $\mathcal{M}$ is typically much more difficult, due to the fact that it might be quite nontrivial to explicitly determine the group $\mathcal{G}$. Indeed, $\mathcal{G}$ arises as a certain subgroup of $\operatorname{Aut}_{\mathrm{R}}^{\omega}(\mathrm{A})$, the group of (relative) symplectomorphisms of the noncommutative symplectic space $\mathbb{A}$ (which can be viewed as a noncommutative space over $\operatorname{Spec}(R)$ ). The later is itself a subgroup of the group $\operatorname{Aut}_{\mathrm{R}}(\mathrm{A})$ of relative superalgebra automorphisms, which is nontrivial to determine in general form (see [21,22]) even in the basic case of a single D-brane sector, for which $A$ is a free superalgebra. Surprisingly, this gives a direct connection between the physics of topological D-branes and certain basic problems in noncommutative algebra, such as the characterization of wild automorphisms. In spite of such difficulties, the space $\mathcal{M}$ has been determined explicitly at least in certain low-dimensional examples [1].

Acknowledgements The author is supported by the European Commision FP6 program MRTN-CT-2004-005104, in which the author is associated with Trinity College Dublin.

\section{References}

[1] C. I. Lazaroiu, "On the non-commutative geometry of topological D-branes", hep-th/0507222

[2] M. Kontsevich, "Formal (non)commutative symplectic geometry.", The Gelfand Mathematical Seminars, 1990_ 1992, 173-187, Birkhäuser Boston, Boston, MA, 1993.

[3] C. I. Lazaroiu, "String field theory and brane superpotentials," JHEP 0110 (2001) 018 |arXiv:hep-th/0107162].

[4] M. Herbst, C. I. Lazaroiu and W. Lerche, "Superpotentials, A(infinity) relations and WDVV equations for open topological strings," JHEP 0502 (2005) 071 |arXiv:hep-th/0402110|.

[5] J. D. Stasheff, "Homotopy associativity of H-spaces, I \& II", Trans. Amer. Math. Soc. 108 (1963), pp. 275-292 and 293-312.

[6] E. Witten, "Mirror manifolds and topological field theory,", in Essays on mirror manifolds, pp 120-158, Internat. Press, Hong Kong, 1992 |arXiv:hep-th/9112056|.

[7] K. Costello, "The A-infinity operad and the moduli space of curves", math.AG/0402015

[8] K. Costello, "Topological conformal field theories and Calabi-Yau categories", math.QA/0412149

[9] H. Kajiura, J. Stasheff, "Homotopy algebras inspired by classical open-closed string field theory", math.QA/0410291

[10] M. R. Gaberdiel and B. Zwiebach, "Tensor constructions of open string theories I: Foundations,” Nucl. Phys. B 505 (1997) 569 |arXiv:hep-th/9705038|.

[11] L. LeBruyn, "Noncommutative geometry @n", unpublished book, available at www.neverendingbooks.org; see also "Three talks on noncommutative geometry@n", math.RA/0312221

[12] V. Ginzburg, "Lectures on Noncommutative Geometry", math.AG/0506603

[13] G.-C. Rota, B. Sagan, P. R. Stein, “A cyclic derivative in noncommutative algebra”, J. of Algebra 64 (1980) 54-75.

[14] D. Voiculescu, “A note on cyclic gradients”, Indiana Univ. Math. J 49 (2000) 3, 837-841. 
[15] W. Crawley-Boevey, P. Etingof, V. Ginzburg, "Noncommutative Geometry and Quiver algebras", math.AG/0502301

[16] M. Van den Bergh, "Double Poisson algebras", math.QA/0410528

[17] V. Ginzburg, "Noncommutative symplectic geometry, quiver varieties and operads", Math. Res. Lett 8 (2001) 3, pp 377-400.

[18] R. Bocklandt, L. Le Bruyn, "Necklace Lie algebras and noncommutative symplectic geometry", Math. Z. 240 (2002) 141-167 [math.AG/0010030|

[19] M. Alexandrov, M. Kontsevich, A. Schwarz, O. Zaboronsky, "The Geometry of the Master Equation and Topological Quantum Field Theory”, Int. J. Mod.Phys. A12 (1997) 1405-1430 |hep-th/9502010|.

[20] A. Hamilton, A. Lazarev, "Homotopy algebras and noncommutative geometry", math.QA/0410621

[21] U. U. Umirbaev, "Tame and wild automorphisms of polynomial algebras and free associative algebras", MaxPlanck Institute Bonn, Preprint MPIM2004-108.

[22] V. Drensky, J.-T. Yu, " The strong Anick conjecture is true", math.RA/0507170

[23] E. Witten, "Chern-Simons gauge theory as a string theory", The Floer memorial volume, 637-678, Progr. Math. 133, Birkhauser, Basel, 1995 |hep-th/9207094|.

[24] V. Ginzburg, "Double derivations and Cyclic homology", math.KT/0505236 